\title{
Morphological changes in capillaries in the ischemic brain in Wistar rats
}

\author{
Yoshiharu Taguchi ${ }^{1}$, Shutaro Takashima ${ }^{1}$, Etsuko Sasahara', Hiroshi Inoue', \\ and Osamu Ohtani ${ }^{2}$
}

'The Second Department of Internal Medicine and ${ }^{2}$ Department of Anatomy, Toyama Medical and

Pharmaceutical University, Toyama, Japan

Summary. The microvasculature in the brain plays a vital role in the maintenance of brain perfusion, and fulfills the dynamic requirements of normal brain functions. It is well known that collateral circulation can be induced by ischemia in cerebral infarctions, but it is not known whether cerebral ischemia affects microvasculatures in the ischemic region. In the present study, we examined quantitatively serial changes in capillaries following bilateral common carotid artery ligation in Wistar rats. After the animals were perfused with tetramethylrhodamine isothiocyanate-labeled gelatin $3 \mathrm{~h}$ $(n=9), 1$ day $(n=9), 7$ days $(n=9)$ and 28 days $(n=9)$ after the ligation, capillary diameters in the brain sections were measured with a confocal laser-scanning microscope. Capillary diameters of the cerebellum did not differ among all groups, while those in the ischemic regions decreased significantly $3 \mathrm{~h}$ after the ligation ( $p<0.01$ ), thereafter gradually returned toward the baseline level, and became significantly larger ( $168 \%$ of the control) 28 days after the ligation $(p<$ 0.01). The density of capillaries in the frontal and parietal cortices increased approximately to 1.3-fold of those of the control level 28 days after the ligation. Transmission electron microscopy showed that the mean ratio of the inner diameter to the outer diameter of capillaries in the frontal cortex became significantly greater 28 days after the ligation $(\mathrm{p}<0.05)$. Our data indicate that capillaries dilate in the ischemic brain region in the chronic phase of cerebral ischemia. It is also suggested that neovascularization occurs in the ischemic brain region.

Received September 15, 2004

Address for correspondence: Dr. Shutaro Takashima, The Second Department of Internal Medicine, Toyama Medical and Pharmaceutical University, Sugitani 2630, Toyama 930-0194, Japan

Tel: +81-76-434-7205; Fax: +81-76-434-5010

E-mail: shutaro@ms.toyama-mpu.ac.jp

\section{Introduction}

In the literature on strokes, much attention has been focused on how to protect ischemic penumbra against neuronal death (Astrup et al., 1981; Fisher and Garcia, 1996). Recently, antithrombotic agents and neuroprotective drugs have been used to improve microcirculation or to prevent neuronal death in patients with ischemic stroke (International Stroke Trial Collaborative Group, 1997; Kawai et al., 1997). However, the effects of these drugs are limited. If an occluded main vessel can not be reopened in the early stage of a stroke, the development of collateral vessels will be crucially important to save ischemic tissues from neuronal death.

We have previously reported that hemispheric hypoperfusion, which was usually detected in patients with watershed infarctions, was recovered in the chronic phase (Takashima et al., 2000). It is well known that collateral circulation can be induced by ischemia in cerebral infarctions as well as in myocardial infarctions (Herz et al., 1998; van Everdingen et al., 1998). However, there are few reports concerning microcirculation in the chronic stage of cerebral ischemia.

In an experimental model, bilateral common carotid artery ligation in Wistar rats severely reduced cerebral blood flow of the forebrain in the early phase (Fujishima et al., 1981; Umehara et al, 1982; Tsuchiya et al., 1992; Otori et al., 2003), which returned to about $70 \%$ of the control value four weeks later (Otori et al., 2003). Additionally, the development of collateral vessels was confirmed in an animal model (Coyle and Heistad, 1987, 1991). Although it has been detected that leptomeningeal vessels become enlarged in various ischemic conditions (Liebskind, 2003), changes in capillaries are still undetermined.

Since capillaries play a vital role in fulfilling the dynamic requirements of brain functions by exchanging substances 
between the blood and the brain tissues (Guyton, 1986), the capillaries might be affected by metabolic damage in various ischemic conditions. Therefore, in the present study, we quantitatively examined the morphological changes in capillaries following bilateral common carotid artery ligation in Wistar rats, using light microscopy, confocal laserscanning microscopy (CLSM), and transmission electron microscopy (TEM).

\section{Materials and Methods}

\section{Surgical operation}

Male Wistar rats (Japan Clea Inc., Tokyo) aged nine to thirteen weeks were used. This study was conducted in accordance with the Guidelines for the Care and Use of Laboratory Animals of Toyama Medical and Pharmaceutical University. The experimental animals were divided into five groups including a sham group. Animals of $3-\mathrm{h}(\mathrm{n}=9), 1-$ day $(n=9)$, 7-day $(n=9)$ and 28-day groups $(n=9)$ were kept with food and water ad libitum for 3 h, 1 day, 7 days and 28 days, respectively, after bilateral common carotid artery ligation. All rats were scheduled to have their capillaries examined at 13 weeks of age. The animals were anesthetized with intraperitoneal sodium pentobarbital $(40 \mathrm{mg} /$ $\mathrm{kg}$ ) and were allowed to breathe spontaneously throughout the surgical procedure. The common carotid arteries of both sides were carefully separated from the cervical sympathetic and vagal nerves through a midline cervical incision, ligated doubly with silk sutures, and completely cut by scissors. The sham group rats $(n=9)$ aged nine weeks underwent the same surgical operation without bilateral common carotid artery ligation. Body temperature was monitored and maintained between $36.5^{\circ} \mathrm{C}$ and $37.5^{\circ} \mathrm{C}$ during the surgical proce- dure. Arterial blood pressure and blood gases were measured at the beginning and the end of each experiment.

\section{Preparation of tetramethylrhodamine isothiocyanate- labeled gelatin}

Tetramethylrhodamine isothiocyanate (TRITC)-labeled gelatin was prepared according to the method introduced by Hashimoto et al (1998). Gelatin from bovine skin (approx. 225 Bloom, No. G9382) was purchased from Sigma (St. Louis, MO, USA) and TRITC (No. 535-07443 REO) from Wako Pure Chemical (Osaka). The reacted mixlure of gelatin and TRITC was dialyzed across a cellulose mem-

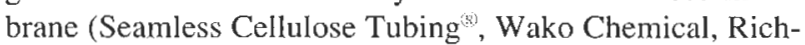
mond, VA, USA) in the dark at $37^{\circ} \mathrm{C}$ against $0.01 \mathrm{M}$ phosphate buffered saline (PBS, pH7.2) containing $0.01 \%$ $\mathrm{NaN}_{3}$. The TRITC-labeled gelatin solution was subsequently stored in the dark at $4^{\circ} \mathrm{C}$.

\section{Perfusion of capillaries with the TRITC-labeled gelatin solution}

Prior to perfusion of the capillaries, the TRITC-labeled gelatin solution was dissolved in a hot water bath $\left(60^{\circ} \mathrm{C}\right)$ and was diluted with PBS to adjust the final concentration to $10 \%$. Under anesthesia with sodium pentobarbital (40 $\mathrm{mg} / \mathrm{kg}$ IP), the TRITC-labeled gelatin solution $\left(37^{\circ} \mathrm{C}\right.$ ) was injected slowly and manually into the ascending aorta using a $10-\mathrm{m} /$ syringe, until the blood no longer overflowed through the right atrium. The brain was rapidly removed and placed in $4 \%$ paraformaldehyde at $4{ }^{\circ} \mathrm{C}$ for 5 days. According to the atlas of the rat brain (Paxison and Watson, 1986), coronal sections ( $200 \mu \mathrm{m}$ thick) -including the frontal cortex, the caudate-putamen, the parietal cortex, the

Table 1. Comparison of mean capillary diameters among the study groups

\begin{tabular}{|c|c|c|c|c|c|}
\hline \multirow{2}{*}{ Region } & \multirow{2}{*}{$\begin{array}{l}\text { Sham group } \\
(N=9)\end{array}$} & \multicolumn{4}{|c|}{ BCAL groups $(\mathrm{N}=9$ each $)$} \\
\hline & & 3-h & 1-day & 7-day & 28-day \\
\hline Frontal cortex & $4.81 \pm 0.24$ & $3.53 \pm 0.25^{* * 4 i}$ & $4.51 \pm 0.20^{*}$ & $4.72 \pm 0.25$ & $8.09 \pm 0.53^{\mathrm{k} * \mathrm{k} \dagger}$ \\
\hline Caudate putamen & $4.58 \pm 0.55$ & $3.40 \pm 0.34 * \%$ & $4.09 \pm 0.28 \dagger$ & $4.21 \pm 0.25$ & $7.97 \pm 0.38 \cdots$ \\
\hline Parietal cortex & $4.76 \pm 0.63$ & $3.65 \pm 0.24 \%+7$ & $4.14 \pm 0.31+$ & $4.68 \pm 0.24$ & $8.61 \pm 0.37 * 6+1$ \\
\hline Thalamus & $4.61 \pm 0.51$ & $3.61 \pm 0.16^{* \%+1}$ & $4.26 \pm 0.17+\div$ & $4.32 \pm 0.36$ & $7.95 \pm 0.41 * *+\phi$ \\
\hline Cerebellum & $4.83 \pm 0.52$ & $4.55 \pm 0.25$ & $4.67 \pm 0.23$ & $4.44 \pm 0.32$ & $4.62 \pm 0.35$ \\
\hline
\end{tabular}

BCAL: bilateral common carotid artery ligation

Data are mean $\pm \mathrm{SD}(\mu \mathrm{m})$, ** $\mathrm{p}<0.01$ versus the sham group, $\mathrm{p}<0.05$ versus the sham group,

if $p<0.01$ versus the cerebellum of the same group 
thalamus, and the cerebullum-were prepared by a vibratome. The sections were collected on glass slides and filled with PBS.

\section{Determination of capillary diameter}

The sections were observed with a Carl Zeiss laser-scanning confocal imaging system 510 (LSM-510, Germany). The laser source was a He-Ne laser with the principal line at $543 \mathrm{~nm}$ (red). The emitted fluorescence was divided with a dichroic mirror at $560 \mathrm{~nm}$. The fluorescence of TRITC was observed through a long pass filter at $590 \mathrm{~nm}$. Laser intensity was set at $100 \%$ of laser power. The laser power, iris, gain, and duration of sampling time were fixed during the acquisition of data in each section. For tissue sections from each group of rats, 3 areas of each region were screened under a $10 \times$ objective lens with a numerical aperture of 0.3 . Areas of interest in each section were scanned in a $512 \times 512$ pixel format in the $x-y$ direction using a $4 \times$ frame-scan average and 20 thin optical slices at a $1 \mu \mathrm{m}$ step-size. A three-dimensional reconstruction image of the vascular bed was obtained with optional LSM-510 software (Carl Zeiss Co.) to determine a capillary network in a given vascular bed. Images obtained under a $40 \times$ objective lens had a resolution of $0.5 \mu \mathrm{m} /$ pixel in the $\mathrm{x}$ and $\mathrm{y}$ dimensions and $1.0 \mu \mathrm{m} /$ pixel along the $\mathrm{z}$-axis. Capillary diameters of each region were measured at 30 points in each animal.

\section{Transmission electron microscopy}

The remaining 5 rats from the 28-day group and of the sham group were used for transmission electron microscopy (TEM) of capillaries. Under deep anesthesia with an intraperitoneal injection of $40 \mathrm{mg} / \mathrm{kg}$ sodium pentobarbital, each animal was perfused with $100 \mathrm{~m} /$ saline containing $1 \mathrm{~m} l$ heparin through a polyethylene tube inserted into the aorta, which was followed by perfusion with $200 \mathrm{~m} l$ half Karnovsky fluid (2\% paraformaldehyde and $2.5 \%$ glutaraldehyde in a $0.1 \mathrm{M}$ phosphate buffer, $\mathrm{pH} 7.38$ ).

Table 2. Comparison of mean densities of capillaries

\begin{tabular}{lcc}
\hline & Sham group & BCAL group \\
\hline Frontal cortex & $3.46 \pm 0.90$ & $4.56 \pm 0.73^{*}$ \\
Parietal cortex & $3.22 \pm 0.38$ & $4.13 \pm 0.51^{*}$ \\
\hline
\end{tabular}

BCAL: 28 days after the bilateral common carotid artery ligation. Data are mean $\pm \mathrm{SD}\left(10^{-4} \mu \mathrm{m}^{-2}\right)$, $* \mathrm{p}<0.05$ versus the sham group
Brains were removed thereafter, and cut by a vibratome into $50 \mu \mathrm{m}$-thick coronal sections including either the frontal cortex or the cerebellum according to the atlas of the rat brain. The sections from the same areas for which we had measured the diameters of capillaries by a CLSM were embedded in Quetol 812 epoxy resin. Sections of $1 \mu \mathrm{m}$ thickness were stained with $1 \%$ toluidine blue for a light microscope, and ultrathin sections were contrasted with uranyl-acetate and lead-citrate and observed in a transmission electron microscope. In order to determine the capillary density, numbers of capillaries were counted in randomly chosen pictures taken with a $40 \times$ objective Iens. The structure of capillary endothelial cells and pericytes was evaluated between the 28-day group and the sham group, and the ratios of the inner diameter to the outer diameter of capillaries in the frontal cortex and the cerebellum were measured in each group.

\section{Statistics}

In each region, the mean diameter of capillaries was compared among all the groups by analysis of variance (ANOVA). In each animal group, the mean diameter of capillaries was compared among all the regions by ANOVA. When this procedure indicated a significant difference, a t-test with the Bonferroni correction was performed for multiple comparisons. The ratio of the inner diameter to the outer diameter of capillaries in each region was compared between two groups by unpaired t-test. All values were expressed as mean \pm SD. A value of $\mathrm{p}<0.05$ was considered statistically significant.

\section{Results}

There were no statistical differences in temperature, mean arterial blood pressure, or blood gas analysis among the animal groups (data not shown).

Mean capillary diameters of the frontal cortex, the caudate-putamen, the parietal cortex, the thalamus, and the cerebellum for all groups are shown in Table 1. In the sham group, there were no statistical differences in the capillary diameters among the five regions (Table 1). The diameters of 1350 capillaries among the five regions were determined in the sham group to obtain a mean diameter of $4.73 \pm 0.48 \mu \mathrm{m}$.

The capillary diameter of the cerebellum did not differ among all the groups; this indicates that the capillary diameter of the cerebellum could serve as the inner control for each study group (Table 1). In contrast, the capillary diameter in the forebrain decreased significantly $3 \mathrm{~h}$ after bilateral common carotid artery ligation $(p<0.01)$, and thereafter gradually returned toward the baseline level as seen in the 

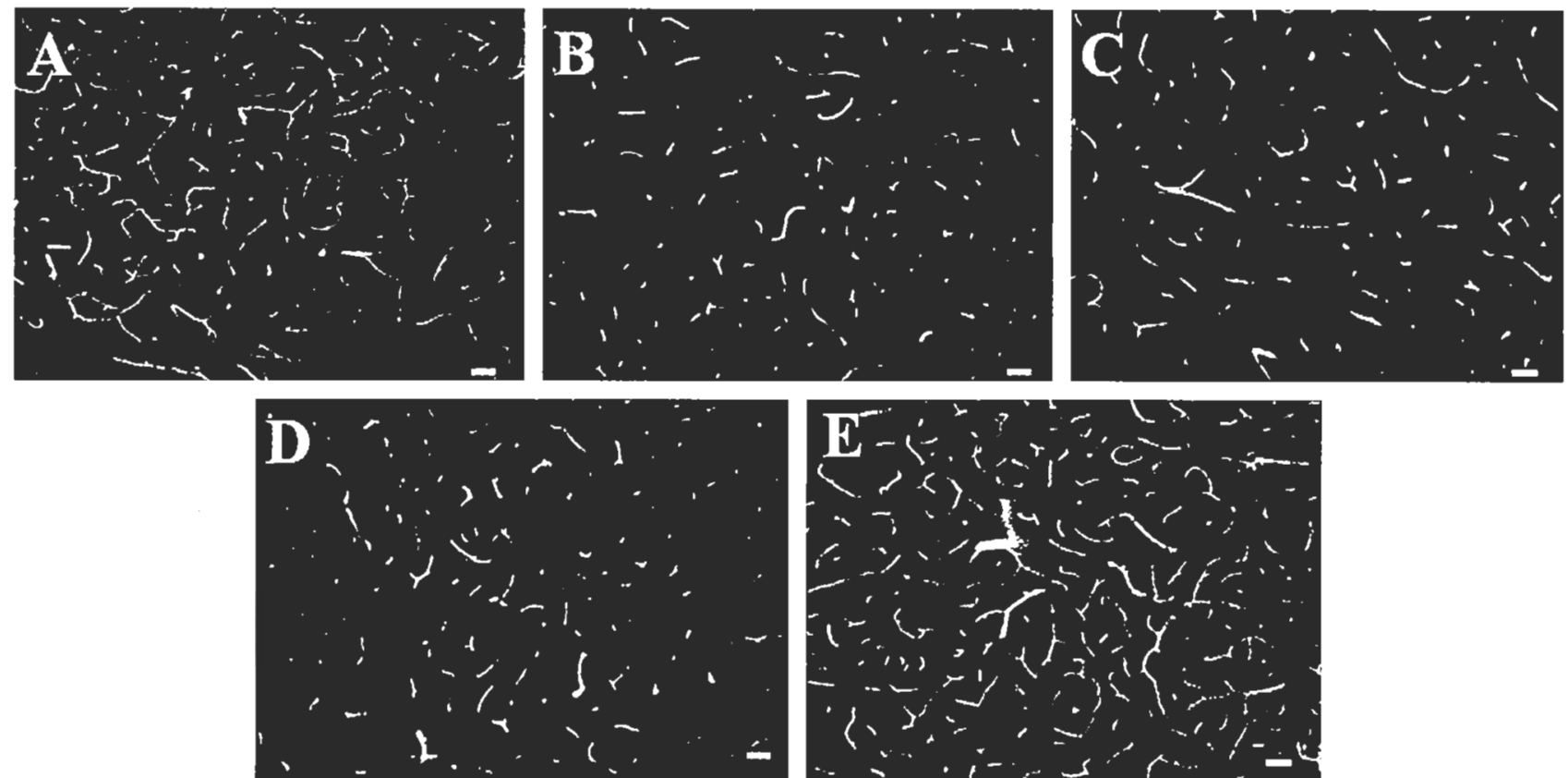

Fig. 1. Confocal laser-scanning micrographs of serial optical sections showing capillaries in the frontal cortex, filled with TRITC-labeled gelatin. A: the sham group, B: the 3-hour group, C: the 1-day group, D: the 7-day group, E: the 28-day group. Scale bars: $25 \mu \mathrm{m}$. The capillary diameters in the frontal cortex decreased slightly from 3 hours (B) to 1 day (C) and 7 days (D) after bilateral common carotid artery ligation. They returned to almost the same level as the sham group (A), and became greatly enlarged 28 days after the ligation.

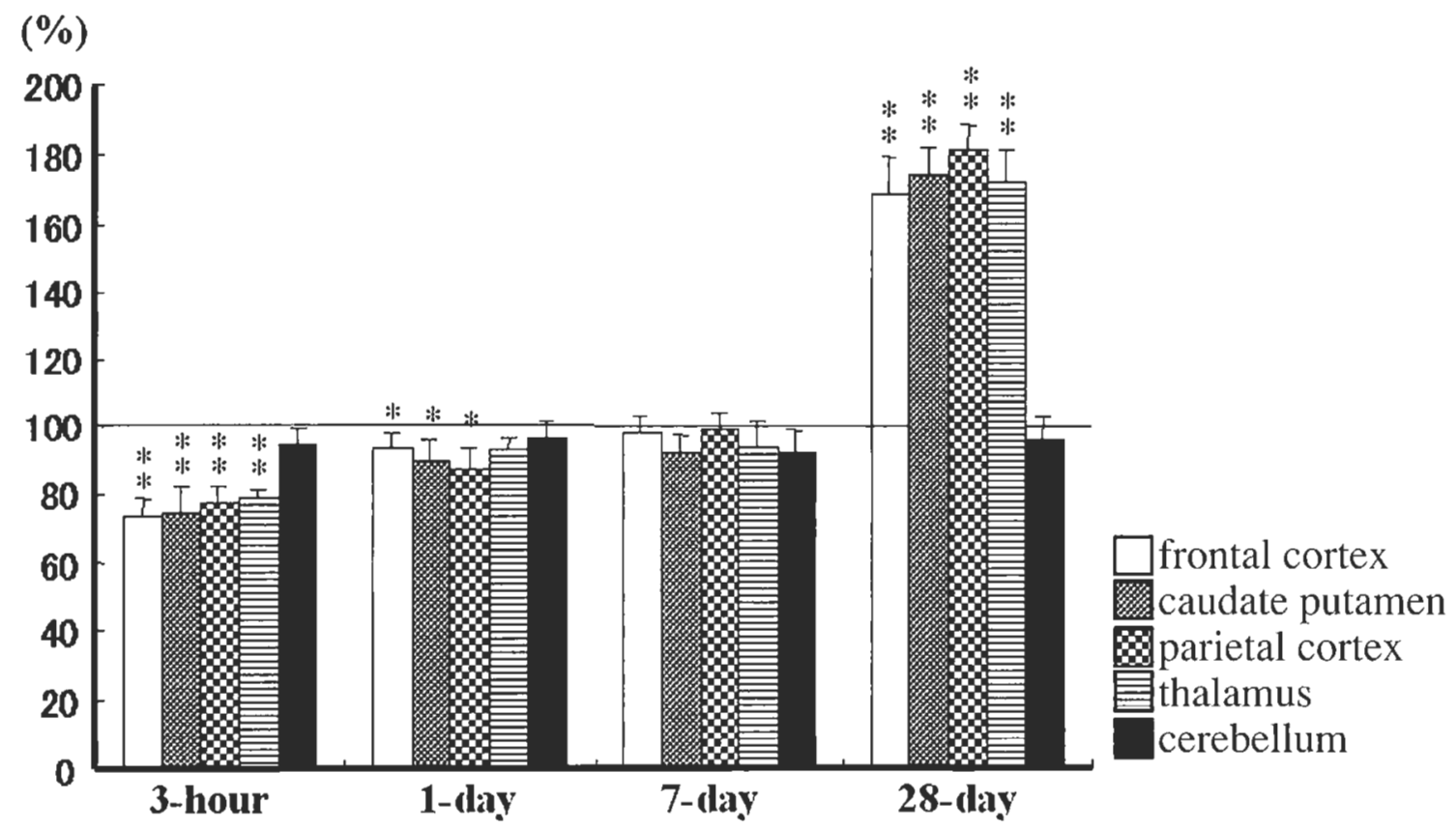

Fig. 2. Serial percentage changes in the capillary diameters, with the control value of the sham group being $100 \%$. $* * \mathrm{p}<0.01$ and $* \mathrm{p}<0.05$ versus the control value. 
Fig. 3. Optical sections showing the cerebral capillaries filled with TRITC-labeled gelatin. A: the frontal cortex of the sham group, B: the frontal cortex of the 28-day group, C: the parietal cortex of the sham group, D: the parietal cortex of the 28-day group, E: the cerebellum of the sham group, $\mathbf{F}$ : the cerebellum of the 28-day group. In the frontal and the parietal cortex, the capillary diamcters of the 28day group (B, D) were greater than those of the sham group $(\mathbf{A}, \mathbf{C})$, while those in the cerebellum did not differ between the two groups $(\mathbf{E}, \mathbf{F})$. Scale bars: $25 \mu \mathrm{m}$
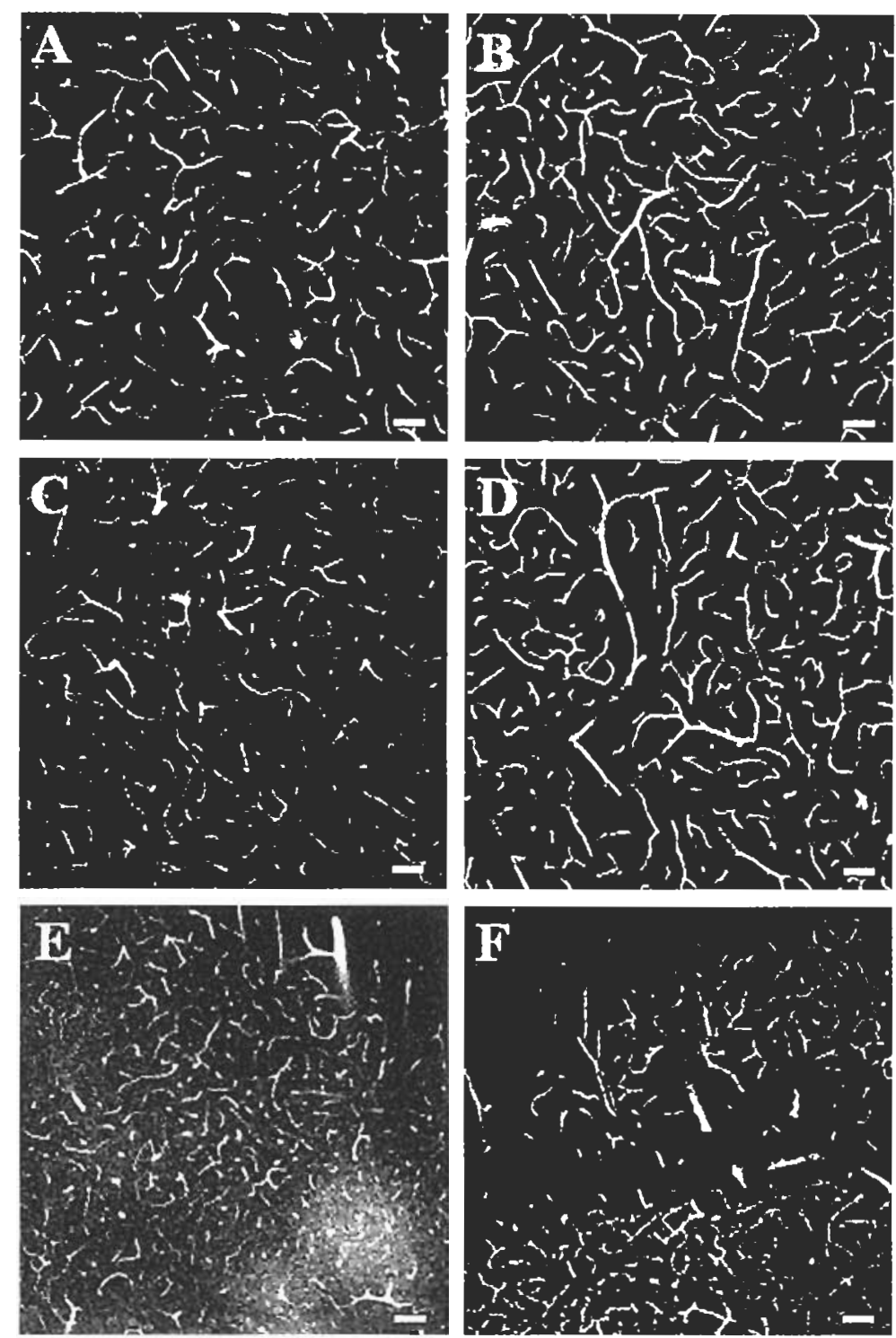

sham group, or toward the control level as seen in the cerebellum (Table 1, Fig. 1, 2). Noteworthily, the capillary diameter in the forebrain became significantly larger (168\% of the control) 28 days after the ligation as compared with the sham group or the inner control in the cerebellum $(\mathrm{p}<$ 0.01 ) (Table 1, Fig. 1-3).

The capillary densities in the frontal and parietal cortices are shown in Table 2. The capillary densities in both frontal and parietal cortices significantly increased to approximately 1.3-fold that of the control level 28 days after the ligation of the bilateral common carotid arteries $(\mathrm{p}<0.05)($ Fig. 4, 5)

Light microscopy showed no infarct lesions in the frontal cortex or in the cerebellum 28 days after bilateral common carotid artery ligation. TEM demonstrated no obvious differences in capillary endothelial cells or pericytes in the frontal cortex and the cerebellum between the 28-day group and the sham group. However, in the frontal cortex, the mean ratio of the inner diameter to the outer diameter of capillaries was significantly greater in the 28-day group than in the sham group ( $\mathrm{p}<0.05$ ) (Fig. 6, 7). The ratio in the cerebellum did not differ between these two groups. 

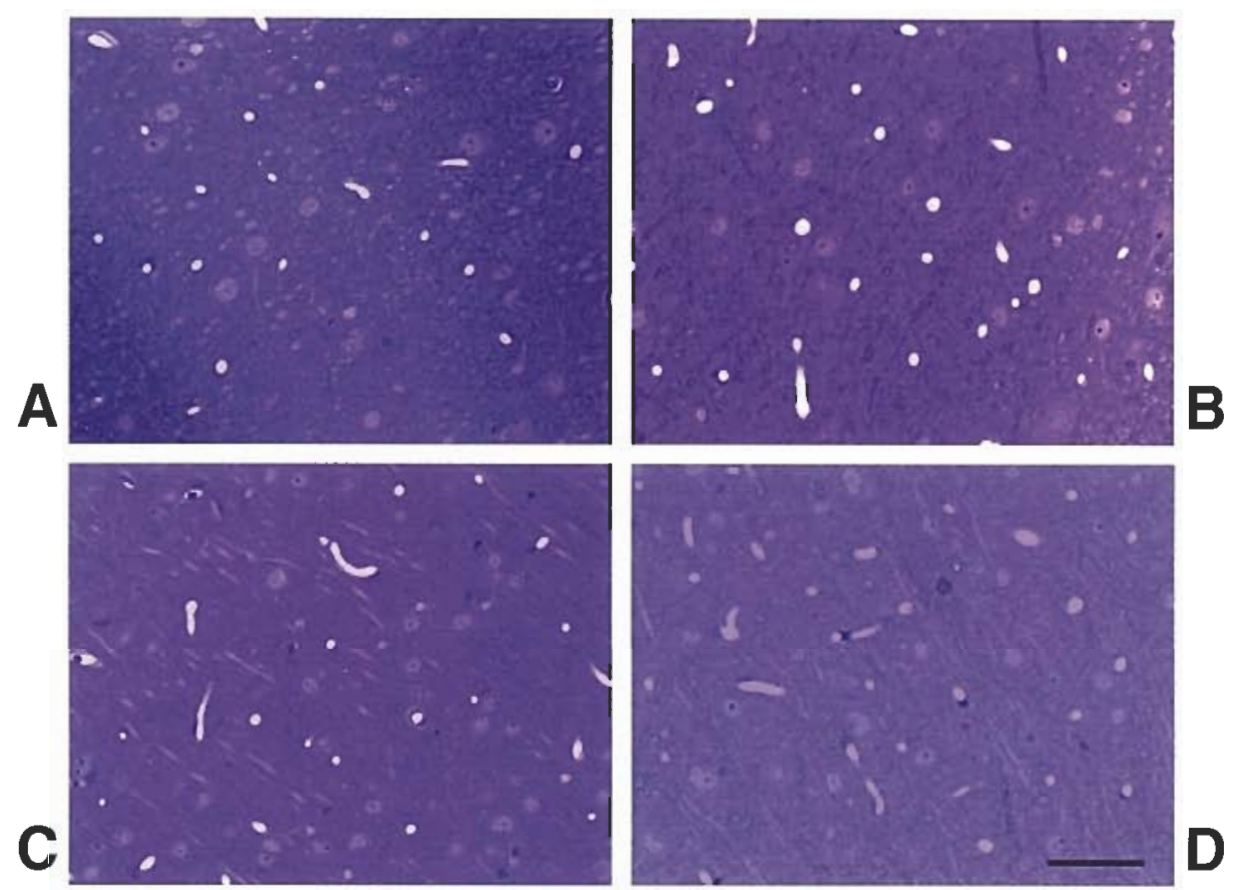

Fig. 4. Light micrographs of the frontal $(\mathbf{A}, \mathbf{B})$ and parietal $(\mathbf{C}, \mathbf{D})$ cortices in the sham group (A, C) and in the 28-day group (B, D). Scale bar: $50 \mu \mathrm{m}$
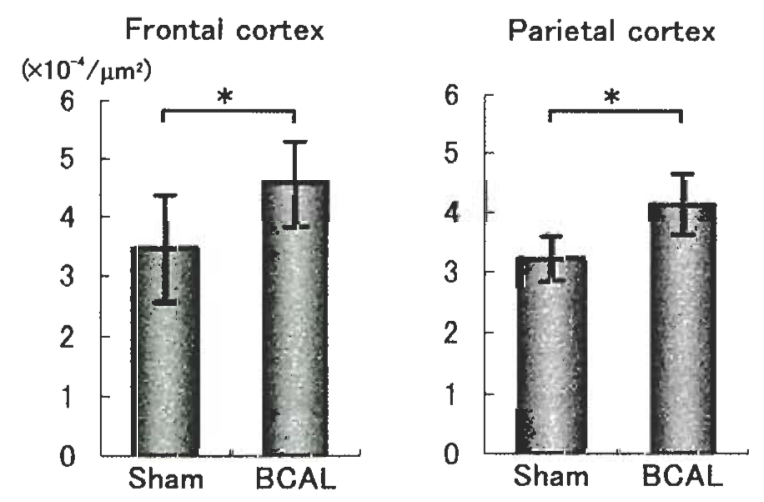

Fig. 5. The capillary densities of the frontal and parietal cortices in the sham group (sham) and in the 28-day group after the ligation of the bilateral common carotid arteries (BCAL). $* \mathrm{p}<0.05$

\section{Discussion}

Major findings of the present study are as follows. The diameter of capillaries in all regions where cerebral ischemia was induced by bilateral common carotid artery ligation decreased slightly from $3 \mathrm{~h}$ to 1 day after the ligation in Wistar rats. Seven days after the ligation, the capillary diameter returned to almost the same dimension as that of the sham group. Four weeks after bilateral common carotid artery ligation, capillaries became greatly enlarged. This is, to our knowledge, the first report that demonstrates the time course of changes in capillary diameter following cerebral ischemia in experimental animals.

We investigated capillaries three-dimensionally under a CLSM, which has the advantage of depth resolution and tissue penetration, and therefore allows serial optical sections and reconstruction of a three-dimensional picture (Dirnagl et al., 1991; Zhang et al., 1999). Morris et al. (2000) measured 8930 capillary diameters in Wistar rats with a CLSM and obtained the mean diameter of $4.15 \pm$ $1.24 \mu \mathrm{m}$. Hudetz et al. (1993) reported the mean capillary diameter was $4.5 \pm 1.1 \mu \mathrm{m}$ using intravital video recordings. The average capillary diameter in the sham-control rats in our study was $4.73 \pm 0.48 \mu \mathrm{m}$, which closely matches the previous results by others. 

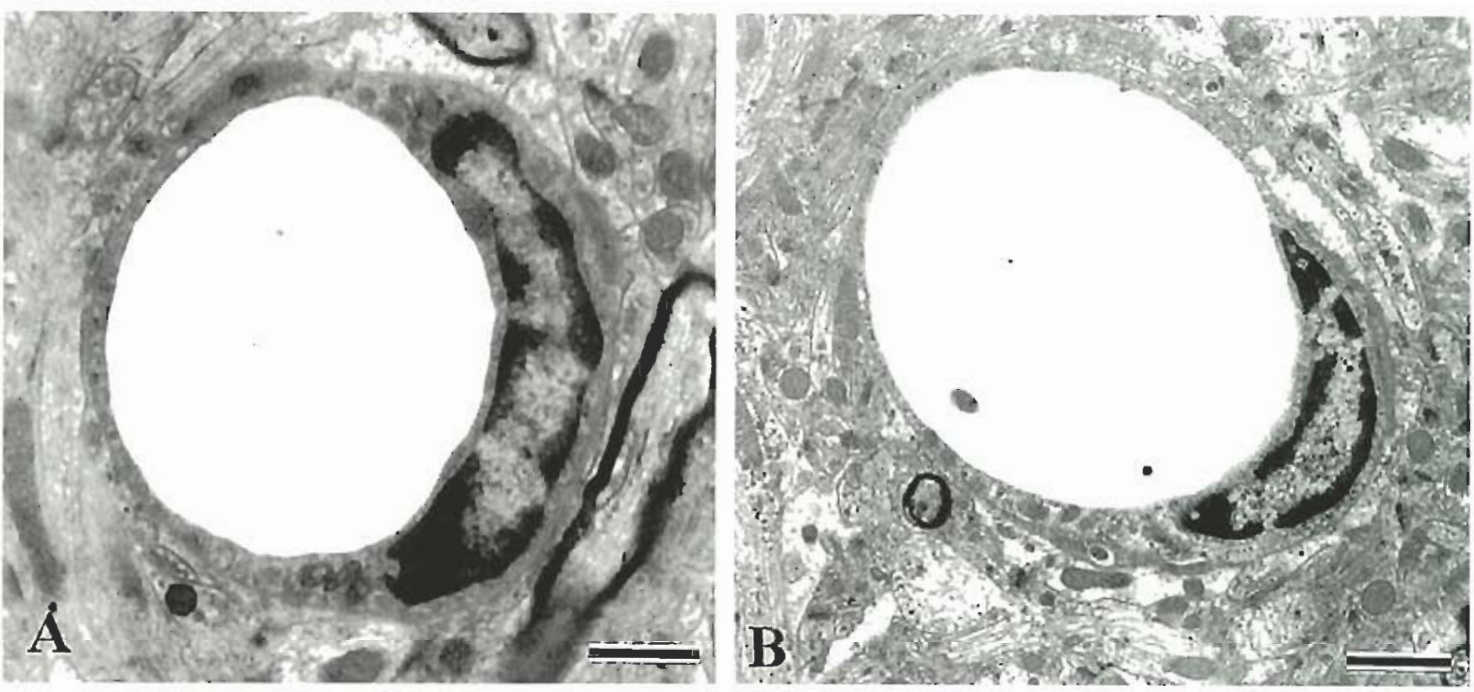

Fig. 6. Transmission electron micrographs of the capillaries in the frontal cortex. A: Sham group, B: BCAL group (28 days after bilateral common carotid artery ligation). Scale bars: $1 \mu \mathrm{m}$

Local control of cerebral blood flow can be divided into two different phases, i.e., acute control and long-term control. In acute control, muscle fibers of metarterioles and precapillary sphincters are controlled by several metabolites in the tissues with resultant changes in regional cerebral blood flow. On the other hand, there may be a continuous reconstruction of the vasculature to meet the needs of tissue metabolism in long-term control (Guyton, 1986). However, there are few reports on changes in capillaries in the setting of long-term control, and it has been believed that capillaries may not dilate by themselves. Boero et al. (1999) examined the capillary nets in the brain of mice exposed to 4 weeks of hypobaric hypoxia, and demonstrated capillaries dilated on chronic hypoxia. The present study demonstrated that capillaries dilate on chronic cerebral hypoperfusion as on chronic hypoxia.

Noteworthily, the capillary diameter in the present study increased to $168 \%$ of the control 4 weeks after the bilateral common carotid artery ligation. Moreover, our TEM showed that the ratio of the inner diameter to the outer diameter of capillaries in the ischemic region significantly increased 4 weeks after the ligation. These facts seem to indicate that the interface between the blood and neuronal tissues became thin and wide, which would be beneficial for exchanging substances between circulating blood and the ischemic tissue. Although the mechanism by which capillaries dilated in our experimental model is not known at this time, the capillary dilatation we observed seems to be a phenomenon to meet the needs of tissue metabolism.

In an experimental ischemic model, Coyle and Panzenbeck (1990) demonstrated that the posterior communicating artery became enlarged, and that collateral vessels through leptomeningeal anastomoses developed following unilateral

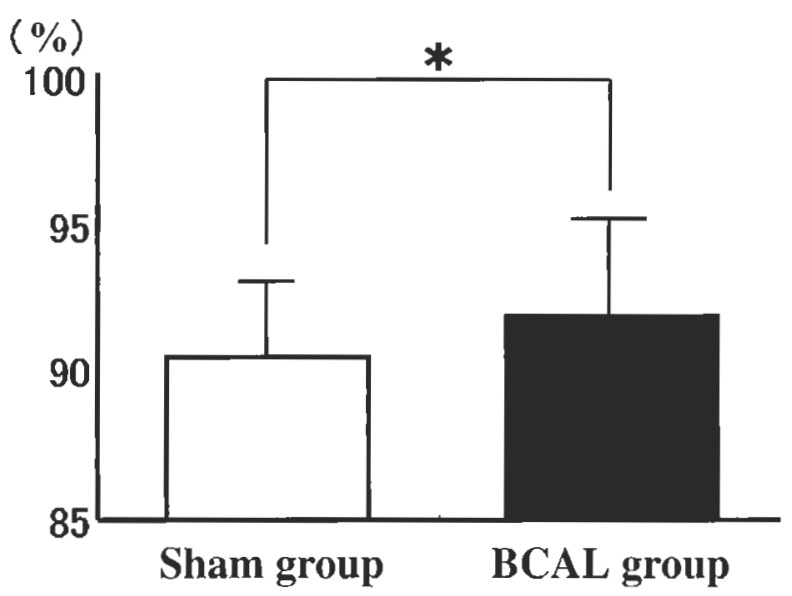

Fig. 7. The ratios of inner diameter to outer diameter of capillaries in the frontal cortex. BCAL group: 28 days after bilateral common carotid artery ligation. * $\mathrm{p}<0.05$ 
internal carotid artery occlusion. Hugo et al. (2000) found a strong increase in the number of newly formed vessels at the border of the infarction 48 to $72 \mathrm{~h}$ after permanent middle cerebral artery occlusion. Furthermore, they showed a strongly up-regulated expression of vascular endothelial growth factor (VEGF) and VEGF receptors (VEGFRs) in the ischemic border of the neocortex (Hugo et al., 2000). The present study showed the density of capillaries in the frontal and parietal cortices increased to some 1.3-fold of that of the control level 28 days after bilateral common carotid artery ligation. This presumably indicates that neovascularization occurred in the ischemic brain regions, as suggested by others (Boero et al., 1999). Although we did not study how this phenomenon occurred, the ischemia perhaps induced an up-regulated expression of VEGF and VEGFRs, as suggested by Hugo et al. (2000).

In the present study, TRITC-labeled gelatin was injected manually, which may affect the diameter of capillaries. However, there were no statistical differences among all groups with regard to the diameter of capillaries of the cerebellum. In addition, the capillary diameter of the forebrain, where perfusion arteries were occluded by bilateral common carotid artery ligation, was significantly greater than that of the cerebellum 28 days after ligation. Furthermore, our light micrographs of the frontal and parietal cortices of the brain without injection of a gelatin tracer definitely showed the dilatation of capillaries 28 days after the ligation (Fig. 4). Therefore, the influence of a manual injection of gelatin on capillary diameter seems to be negligible.

In conclusion, the present study has shown that the capillaries dilate remarkably in the ischemic brain region in the chronic phase of cerebral ischemia. This study also suggests that neovascularization occurs in the ischemic brain. Further investigation is required to reveal the mechanisms for the capillary dilatation and neovascularization in chronic cerebral ischemia.

\section{References}

Astrup J, Siesjo BK, Symon L: Thresholds in cerebral ischemia: the ischemic penumbra. Stroke 12: 723-725 (1981).

Boero JA, Ascher J, Arregui A, Rovainen C, Woolsey TA: Increased brain capillaries in chronic hypoxia. $J$ Appl Physiol 86: 1211-1219 (1999).

Coyle P, Heistad DD: Blood flow through cerebral collateral vessels one month after middle cerebral artery occlusion. Stroke 18: 407-411 (1987).
Coyle P, Heistad DD: Development of collaterals in the cerebral circulation. Blood Vessels 28: 183-189 (1991).

Coyle P, Panzenbeck MJ: Collateral development after carotid artery occlusion in Fischer 344 rats. Stroke 21: 316-321 (1990).

Dirnagl U, Villringer A, Gebhardt R, Haberl RL, Schmiedek P, Einhaupl KM: Three-dimensional reconstruction of the rat brain cortical microcirculation in vivo. J Cereb Blood Flow Metab 11: 353-360 (1991).

Fisher M, Garcia JH: Evolving stroke and the ischemic penumbra. Neurology 47: 884-888 (1996).

Fujishima M, Ishitsuka T, Nakatomi Y, Tamaki K, Omae $\mathrm{T}$ : Changes in local cerebral blood flow following bilateral carotid occlusion in spontaneously hypertensive and normotensive rats. Stroke 12: 874-876 (1981).

Guyton AC: Textbook of medical physiology, 7th ed, W.B. Saunders, Philadelphia, 1986.

Hashimoto H, Ishikawa H, Kusakabe M, Simultaneous observation of capillary nets and tenascin in intestinal villi. Anat Rec 250: 488-492 (1998).

Herz RCG, Hillen B, Versteeg DHG, De Wildt DJ: Collateral hemodynamics after middle cerebral artery occlusion in Wistar and fischer-344 rats. Brain Res 793: 289296 (1998).

Hudetz AG, Greene AS, Feher G, Knuese DE, Cowley $\mathrm{AW}$ : Imaging system for three-dimensional mapping of cerebrocortical capillary networks in vivo. Microvasc Res 46: 293-309 (1993).

Hugo J, Marti H, Bernaudin M, Bellail A, Schoch H, Euler M, Petit Em Risau W: Hypoxia-induced vascular endothelial growth factor expression precedes neovascularization after cerebral ischemia. Am J Pathol 156: 965-976 (2000).

International Stroke Trial Collaborative Group: The international stroke trial (IST): a randomised trial of aspirin, subcutaneous heparin, both, or neither among 19435 patients with acute ischemic stroke. Lancet 349 : 1569-1581 (1997).

Kawai H, Nakai H, Suga M, Yuki S, Watanabe T, Saito K: Effect of a novel free radical scavenger, MCI-186, on ischemic brain damage in the rat distal middle cerebral artery occlusion model. J Pharmacol Exp Ther 281: 921 927 (1997).

Liebeskind DS: Collateral circulation. Stroke 34: 22792284 (2003).

Morris DC, Davies K, Zhang Z, Chopp M: Measurement of cerebral microvessel diameters after embolic stroke in rat using quantitative laser scanning confocal microscopy. Brain Res 876: 31-36 (2000). 
Otori T, Katsumata T, Muramatsu H, Kashiwagi F, Katayama $Y$, Terashi A: Long-term measurement of cerebral blood flow and metabolism in a rat chronic hypoperfusion model. Clin Exp Pharmacol Physiol 30: 266-272 (2003).

Paxison G, Watson C: The rat brain in stereotaxic coordinates. Academic Press, Orland, 1986.

Takashima S, Taguchi $Y$, Inoue $H$, Hirade S, Nagahori T: A profound hemispheric hypoperfusion with relatively small infarction indicatcs a progressing stroke. Keio Med J 49: A77-A79 (2000).

Tsuchiya M, Sato K, Yura S, Yonemasu Y: Cerebral blood flow and histopathological changes following permanent bilateral carotid artery ligation in Wistar rats. Exp Brain Res 89: 87-92 (1992).
Umehara S, Yoshida T, Iwayama K, Mabe H, Nagai H: Experimental study of ischemic rat brain: correlation of pathological findings with rCBF. Brain Nerve 34: 179185 (1982).

van Everdingen KJ, Visser GH, Klijn CJM, Kappelle LJ, van der Grond J: Role of collateral flow on cerebral hemodynamics in patients with unilateral internal carotid artery occlusion. Ann Neurol 44: 167-176 (1998).

Zhang Z, Davies K, Prostak J, Fenstermacher J, Chopp M: Quantitation of microvascular plasma perfusion and neuronal microtubule-associated protein in ischemic mouse brain by laser-scanning confocal microscopy. J Cereb Blood Flow Metab 19: 68-78 (1999). 